\title{
The Pattern of Allopurinol Prescription Among Chronic Kidney Disease Patients in a Tertiary Care Centre: A Single-Centre Experience
}

This article was published in the following Dove Press journal: International Journal of General Medicine

\author{
Sami Alobaidi $\mathbb{D}^{\prime}$ \\ Naji Dwid (D) ${ }^{2}$ \\ Khaldoun Shikh Souk ${ }^{3}$ \\ Mohamed Cheikh (D) ${ }^{4}$ \\ Ahmed Mandurah ${ }^{3}$ \\ Khaled Al-Khatib ${ }^{3}$ \\ Ans Ahmed (iD ${ }^{3}$ \\ Hani Almoallim (1D ${ }^{5}$ \\ 'Department of Medicine, University of \\ Jeddah, Jeddah, Saudi Arabia; ${ }^{2}$ Doctor \\ Soliman Fakeeh Hospital, Nephrology \\ Fellow for Saudi Commission for Health \\ Specialties, Jeddah, Saudi Arabia; ${ }^{3}$ Doctor \\ Soliman Fakeeh Hospital, Jeddah, Saudi \\ Arabia; ${ }^{4}$ Doctor Soliman Fakeeh Hospital, \\ Rheumatology Fellow for Saudi \\ Commission for Health Specialties, \\ Jeddah, Saudi Arabia; ${ }^{5}$ Medical College, \\ Umm Alqura University (UQU), Makkah, \\ 2144I, Saudi Arabia
}

Context: Hyperuricemia is prevalent in patients with chronic kidney disease (CKD). Although it is associated with CKD incidence and progression, treating asymptomatic hyperuricemia with uric acid-lowering agents is still debatable.

Aim of Work: determine the rate of non-classical prescription of allopurinol in CKD patients. Settings and Design: This was a retrospective study of adult patients prescribed allopurinol with CKD (stages 2-5) in Doctor Soliman Fakeeh Hospital (DSFH) Jeddah, Saudi Arabia, from $1 / 1 / 2016$ to $1 / 1 / 2017$.

Subjects and Methods: Eligible patients were identified from the hospital's pharmacy system and cross-referenced with the electronic health records. Demographic data, laboratory results and indication as recorded by the prescribing physician were extracted. Prescriptions with no indication were categorized based on the uric acid levels. Hyperuricemia was documented as mild $(6-10 \mathrm{mg} / \mathrm{dL}$ in females and $7-13 \mathrm{mg} / \mathrm{dL}$ in males) and severe $(>13 \mathrm{mg} / \mathrm{dL}$ in men and $>10 \mathrm{mg} / \mathrm{dL}$ in women).

Statistical Analysis Used: Descriptive statistics (frequencies, percentages).

Results: From the 594 identified patients, 464 (78.1\%) were males. A third of prescriptions (209/594) had no indication, $43.5 \%$ of which (91/209) had no documented uric acid levels, and $16.3 \%(34 / 209)$ had normal levels. Including patients with undocumented indication, $64.2 \%$ (381/594) were prescribed allopurinol for hyperuricemia, $86.4 \%$ of which $(329 / 381)$ had mild hyperuricemia, and only $13.6 \%$ (52/381) had severe hyperuricemia. Other indications included malignancy-related disorders $(6.2 \%, 37 / 594)$, gouty arthritis $(5.2 \%, 31 / 594)$, and stones of unknown aetiology $(3.4 \%, 20 / 594)$.

Conclusion: The percentage of allopurinol prescription to patients with CKD without a clear indication in our centre was markedly high. This might increase the risk for side effects with no evidence-based benefits.

Keywords: allopurinol, gout, hyperuricemia, hyperuricosuria, over prescription, chronic kidney disease

\section{Introduction}

Around 697.5 million people are living with Chronic Kidney Disease (CKD) worldwide. ${ }^{1}$ It is estimated to affect $9-13 \%$ of the world population. ${ }^{1,2}$ In 2017 , CKD-related mortality represented $4.6 \%$ of all mortalities, ranking as the 12 th leading cause of death worldwide, and reaching as high as the second leading cause of death in some countries. ${ }^{1}$

Uric acid is the metabolic end product of purines, both exogenous from dietary proteins and endogenous from dying or degraded cells. Since two-thirds of the uric
Department of Medicine, University of Jeddah, Jeddah, Saudi Arabia

Email salobaidi@uj.edu.sa 
acid are cleared by the kidneys, hyperuricemia is a common laboratory abnormality in CKD patients. ${ }^{3}$ However, hyperuricemia is also considered an independent predictor for the development of CKD especially in patients with type 2 diabetes mellitus. ${ }^{4-7}$ It is also strongly associated with the risk of CKD progression..$^{8-13}$ This has raised the question of whether hyperuricemia is a causative factor for CKD incidence and progression or merely an association of deteriorating kidney function, or both. In spite of that, treatment of asymptomatic hyperuricemia in CKD patients has been controversial with studies showing conflicting results of the benefit of using uric acidlowering agents to achieve normal uric acid levels. ${ }^{4,14,15}$ Recent published studies have found no added benefit of treating those patients. ${ }^{16,17}$

There is ongoing observation of clinicians prescribing allopurinol without a valid indication in patients with or without CKD. Although side effects of allopurinol are rare, they include allopurinol hypersensitivity reaction, Steven-Jonson Syndrome (SJS), and Toxic Epidermal Necrolysis (TEN) which can be fatal. CKD and Cardiovascular disease, which is fairly prevalent among these patients increases the risk of these side effects. ${ }^{18,19}$ In this study, we aim to assess the prescribing habits of physicians for allopurinol in CKD patients in our centre.

\section{Subjects and Methods}

This was a retrospective descriptive study of all adult patients who have CKD (stages 2-5) and had been prescribed allopurinol in Doctor Soliman Fakeeh Hospital (DSFH) Jeddah, Saudi Arabia, during the period of 1/1/ 2016 till 1/1/2017.

Eligible patients were identified from examining all prescriptions of allopurinol (both in generic and brand names such as Loric, Zyloric, and No-Uric) from the hospital's pharmacy system, and then cross-referencing these with the electronic health records to identify patients with CKD stages 2-5. Patients with CKD stage 1 were excluded from the study as the definition of CKD stage 1 is broad and we could not rely on the available information in our database to identify them. Patient demographic data, laboratory results and relevant indication as recorded by the prescribing physicians' notes were then extracted.

Our pharmacy system does not mandate physicians to specify a diagnosis for each newly prescribed medication or during medications refill. In addition, some medications tend to be re-prescribed without the physicians confirming the indication, especially in medication refill clinics where patients have been on these drugs for a while. Nonphysicians are not allowed to order or reorder medications.

All indications for prescribing allopurinol were confirmed with the hospital records and laboratory results. Prescriptions with no indication were categorized based on the uric acid levels. Hyperuricemia was documented as mild $(6-10 \mathrm{mg} / \mathrm{dL}$ in females and $7-13 \mathrm{mg} / \mathrm{dL}$ in males) and severe $(>13 \mathrm{mg} / \mathrm{dL}$ in men and $>10 \mathrm{mg} / \mathrm{dL}$ in women).

Valid indications were considered only if: significant hyperuricemia $(>13 \mathrm{mg} / \mathrm{dL}$ in $\mathrm{men}$ and $>10 \mathrm{mg} / \mathrm{dL}$ in women), gout, hyperuricosuria of more than $1100 \mathrm{mg} / \mathrm{day}$, kidney stones, malignancy, and haemolysis. The following indications were considered not valid: no documented indication, insignificant hyperuricemia $(<13 \mathrm{mg} / \mathrm{dL}$ in men and $<10 \mathrm{mg} / \mathrm{dL}$ in women).

Descriptive statistics (mean, percentage, and standard deviation) for continuous variables and frequencies for categorical variables were calculated using the Statistical Package for Social science (SPSS) version 25 software program. The study was granted ethical approval from the institutional review board of DSFH. Informed patient consents were not required by the IRB for this retrospective chart review study.

\section{Results}

Our study population consisted of 594 adult patients with CKD stages 2-5 who had a prescription for allopurinol. The mean age was $60 \pm 14$ years. Out of these patients, $464(78.1 \%)$ were males and $130(21.9 \%)$ were females, with male-to-female ratio $4: 1$. The mean duration of use of allopurinol, from first documented prescription until 1/1/ 2017 , was $2.3 \pm 2.2$ years.

Two-hundred and thirty-three patients were in CKD stage $3(39.2 \%)$, followed by 178 patients in stage 2 (30.0\%). CKD stage 5 patients were 116 (19.5\% of all patients), $65.5 \%$ of which (76/116) were on renal replacement therapy (RRT). Stage 4 was the least representative stage in our sample with only 67 patients (11.3\%) (Table 1).

Two-hundred and nine prescriptions (35.2\%) had no documented indication, $43.5 \%$ of which $(91 / 209)$ had no documented uric acid level before the first prescription, and 16.3\% (34/209) had normal uric acid levels. Including patients with undocumented indication but abnormal uric acid levels, 381 out of 594 (64.2\%) were prescribed allopurinol for hyperuricemia; $86.4 \%$ of which $(329 / 381)$ had mild hyperuricemia, and only $13.6 \%(52 / 381)$ had severe hyperuricemia. Thirty-seven of all our patients $(6.2 \%)$ had 
Table I Number of Patients According to CKD Stages

\begin{tabular}{|l|c|c|}
\hline CKD Stages & Frequency & Percent \\
\hline 2 & 178 & 30.0 \\
3 & 233 & 39.2 \\
4 & 67 & 11.3 \\
5 (not on RRT) & 40 & 6.7 \\
5 (on RRT) & 76 & 12.8 \\
Total & 594 & 100.0 \\
\hline
\end{tabular}

Abbreviation: RRT, renal replacement therapy.

Table 2 Indications for Allopurinol Prescription in CKD Patients

\begin{tabular}{|l|c|c|c|}
\hline Indications & Frequency & Percent & Cumulative \% \\
\hline $\begin{array}{l}\text { No indication, no uric } \\
\text { acid levels prior to } \\
\text { prescription }\end{array}$ & 91 & 15.3 & 15.3 \\
$\begin{array}{l}\text { No indication, normal } \\
\text { uric acid levels* }\end{array}$ & 34 & 5.7 & 21 \\
Mild hyperuricemia $^{\dagger}$ & 329 & 55.4 & 76.4 \\
$\begin{array}{l}\text { Severe hyperuricemia } \\
\text { Kidney stone (was not }\end{array}$ & 52 & 8.8 & 85.2 \\
analysed) & 20 & 3.4 & 88.6 \\
Malignancy & 31 & 6.2 & 94.8 \\
Gouty arthritis (not & 594 & 100.0 & 100 \\
confirmed) & & 5.2 & \\
Total & 594 & \\
\hline
\end{tabular}

Notes: *Normal value is up to $6 \mathrm{mg} / \mathrm{dL}$ in females and $7 \mathrm{mg} / \mathrm{dL}$ in males; ${ }^{\dagger}$ mild is up to $10 \mathrm{mg} / \mathrm{dL}$ in female and $13 \mathrm{mg} / \mathrm{dL}$ in males; ${ }^{\ddagger}$ severe is above $10 \mathrm{mg} / \mathrm{dL}$ in females and above $13 \mathrm{mg} / \mathrm{dL}$ in males.

allopurinol prescription for malignancy-related disorders and 31 (5.2\%) had gouty arthritis. An indication for stones of unknown aetiology was found in $20(3.4 \%)$ of all prescriptions of allopurinol (Table 2).

Allopurinol was prescribed without a valid indication (no indication with uric acid levels not checked or normal prior to prescription, mild hyperuricemia) in 454 patients (76.4\%) (Table 2).

\section{Discussion}

In our study, $35.2 \%$ of the prescriptions did not have an indication, although some of these patients had abnormal uric acid levels. In general, asymptomatic hyperuricemia was the main indication to start allopurinol in $64.5 \%$ of patients with CKD, where $86 \%$ of them had mild asymptomatic hyperuricemia. Other documented indications included malignancy-related disorder $(6.2 \%)$, gouty arthritis (5.2\%) and stones of unknown composition (3.4\%). Overall, $76.4 \%$ of all prescriptions did not have a valid indication.
Asymptomatic hyperuricemia is defined as an elevation in serum uric acid concentration in the absence of either symptoms or signs of monosodium urate crystal deposition disease, such as gout or uric acid renal disease. ${ }^{20}$ Although there is a clear strong relationship between hyperuricemia and $\mathrm{CKD}$, the association between hyperuricemia and progression of kidney disease is still controversial. ${ }^{21}$ Although evidence is scarce, it's been initially suggested that asymptomatic hyperuricemia is of no clinical importance until serum uric acid levels exceed at least $13 \mathrm{mg} / \mathrm{dL}$ (773 micromole/L) in men and $10 \mathrm{mg} / \mathrm{dL}$ (595 micromole/L) in women, ${ }^{22}$ and indications for treatment are limited to a prophylaxis of gout and stones. ${ }^{21}$ However, recent small studies have suggested $<6-6.5 \mathrm{mg} / \mathrm{dl}$ for which uric acid might be considered a risk factor for CKD progression. ${ }^{23,24}$

Hyperuricemia is highly prevalent in patients with CKD. Chonchol et al found a strong association between baseline uric acid levels and baseline kidney function. Even individuals with uric acid levels $5.9 \mathrm{mg} / \mathrm{dL}$ to $6.9 \mathrm{mg} / \mathrm{dL}$ had $47 \%$ greater risk of estimated glomerular filtration rate (GFR) decline $\geq 3 \mathrm{~mL} / \mathrm{min}$ per $1.73 \mathrm{~m}^{2}$ annually compared to patients with levels less than $4.40 \mathrm{mg} / \mathrm{dL}^{25}$ although there was no significant association between uric acid level and incidence of CKD. ${ }^{15}$ In a different study, $66 \%$ of patients with non-diabetic stages 3-4 CKD had serum uric acid greater than $7 \mathrm{mg} / \mathrm{dL} .{ }^{26} \mathrm{In}$ patients with gout, $16 \%$ of males and $31.4 \%$ of females had CKD stage $3-5 .^{27}$

The unnecessary use of allopurinol to treat hyperuricemia in CKD patients that was observed in this study could be attributed to many factors. One of them is probably the presence of multiple studies that have shown the association of hyperuricemia and CKD. ${ }^{4,5,7,25}$ Mild hyperuricemia is strongly associated with the risk of CKD in patients with type 2 diabetes ${ }^{4}$ and independently increase the risk for new-onset kidney disease. ${ }^{5}$ In a meta-analysis of 13 observational studies which involved more than 190,000 patients, hyperuricemia was an independent predictor for the development of $\mathrm{CKD},{ }^{7}$ though the question of whether the empiric use of allopurinol in these patients can delay this risk was not examined in this study. Other epidemiological studies showed no relationship between hyperuricemia and the progression of kidney disease. ${ }^{26,28}$ In the Modification of Diet in Renal Disease study (MDRD), 838 patients with CKD stage 3-4 were followed for 10 years, and hyperuricemia was not associated with the development of end-stage renal disease (ESRD). ${ }^{26}$ In the Mild to Moderate Kidney Disease (MMKD) study, non-diabetic 
CKD patients were followed for a 7 year period, and hyperuricemia was also not an independent predictor of CKD progression. ${ }^{28}$ These results argue against the natural tendency among some clinicians to prescribe allopurinol empirically to asymptomatic hyperuricemia in CKD as shown in our study.

It is still unclear how allopurinol or other uratelowering therapies will slow the progression of CKD. Uric acid significantly increases production of reactive oxygen species and angiotensin II which lead to endothelial dysfunction and development of systemic and glomerular hypertension ${ }^{29,30}$ which results in kidney damage. In the tubular cells, uric acid is a key contributor to the development of renal fibrosis in CKD by inducing epithelial to mesenchymal transition. ${ }^{31}$ We assume that clinicians are trying to prevent renal disease progression by all means, including empiric prescription of allopurinol. Again, until now, there is no unified theory of the mechanism of preventing renal disease progression from the perspective of improving serum uric acid levels. The antioxidant effect of allopurinol on the vascular endothelium has shown some benefit on the literature. A metaanalysis published on 2018 which included three RCTs that looked into CKD patients showed a statistically significant benefit on patient with congestive heart failure but a marginally statistical significance benefit on patient with CKD. $^{32}$

Clinical studies also report mixed findings. Results of three meta-analyses, which included most available randomized trials, were not conclusive. ${ }^{33-35}$ Two of the published meta-analyses ${ }^{34,35}$ showed favourable results on using allopurinol. Kanji et al in 2015 included 19 RCTs, treatment with allopurinol in asymptomatic hyperuricemia was associated with significant reductions in serum uric acid levels and a favourable influence on blood pressure and on eGFR compared with untreated controls. Many of the included trials were small, single-centre studies with relatively short duration of follow-up. ${ }^{34}$ The second metaanalysis, which had a significant overlap in the randomized trials with the first one, involved 16 randomized trials with a total of 1211 patients with CKD, uratelowering therapy improved kidney outcomes and reduced the risk of cardiovascular events in adults with CKD and asymptomatic hyperuricemia. ${ }^{35}$ This meta-analysis is limited due to the included studies being of low or very low quality with sample sizes, low number of events, having a considerable proportion of missing baseline patient characteristics, and displaying heterogeneity in baseline kidney function. In contrast, a meta-analysis by Bose et al in 2014, which involved 8 trials of patients with or without CKD at baseline, reported that allopurinol therapy had no effect on renal function in most of the RCTs but showed a reduction of serum creatinine levels in some studies. ${ }^{33}$

Since the previous meta-analyses, three large randomized controlled clinical trials addressed the use of uratelowering therapy for the prevention of kidney disease progression. The FEATHER study, which was published in 2018, showed that Febuxostat did not slow the progression stage 3 CKD and asymptomatic hyperuricemia in Japanese patients. ${ }^{36}$ The Preventing Early Renal Loss in Diabetes (PERL) trial ${ }^{37}$ investigated the use of allopurinol in patients with type 1 diabetes mellitus and mild CKD (CKD stages 1 to 3 ). The measured glomerular filtration rate after 3 years did not differ significantly between the two groups although the serum uric acid levels in both groups were not significantly high $(6.1 \pm 1.5 \mathrm{mg} / \mathrm{dl})$. The Controlled Trial of Slowing of Kidney Disease Progression from the Inhibition of Xanthine Oxidase $(\mathrm{CKD}-\mathrm{FIX})^{16}$ included patients who had stage 3 or 4 CKD with a rapid decline in the estimated GFR or clinically significant proteinuria at baseline. As in the PERL trial, treatment with allopurinol had no significant effect on the rate of GFR decline. The neutral results of these studies indicate that it is unlikely that lowering serum urate level would slow the progression of CKD, although some groups of patients, such as the younger population with early $\mathrm{CKD}$, require further evaluation. ${ }^{38}$

The main limitation of this study is that our data depended on the doctors' documentation on the EHR, which was not always complete. As such, we could not comment on the causes of CKD, patients' doses, compliance, or side effects. This was also the main reason for being unable to include patients with CKD stage 1 . In addition, further studies that explore the management of patients with asymptomatic hyperuricemia and the reasons for prescribing allopurinol or withholding it would be invaluable in understanding the true magnitude of the dilemma.

In conclusion, allopurinol was prescribed for CKD patients in our facility without clear and valid indications in $76.4 \%$ of the cases. This exposes patients to the risk of developing serious side effects without adding evidencebased benefits. Physicians should apply basic principles of clinical reasoning before prescribing any drug, including allopurinol. 


\section{Key Messages}

- Current evidence does not support use of allopurinol in patients with CKD for asymptomatic hyperuricaemia.

- Prescribing should be evidence based and always incorporate a documented indication.

- Local practice should be audited regularly, especially when unnecessary prescriptions carry risks of side effects.

\section{Ethics Statement}

The IRB did not ask for informed patient consents. This is based on the initial consent that is signed by every patient who is seen in the hospital whether inpatient or outpatient that states that his data can be utilized. The IRB believe that the analysis that we did is a retrospective one where usually consent from every single patient is not required. This is supported by the fact that we are not using any data to identify individual patient on the analysis of the patients. Usually retrospective chart review of hospital records does not require individualized consent from patients as long as the IRB approve the original study design. We believe that we are fulfilling the Helsinki research protocol as we are not using any kind of intervention affecting patient safety and patient privacy was preserved.

\section{Acknowledgment}

We would like to show our appreciation to Al Zaidi Chair of Research in Rheumatic Diseases (ZCRD) for supervising and funding this project.

\section{Author Contributions}

All authors contributed to data analysis, drafting or revising the article, have agreed on the journal to which the article will be submitted, gave final approval of the version to be published, and agree to be accountable for all aspects of the work.

\section{Disclosure}

Prof. Dr. Hani Almoallim report partial funding and logistic support from Alzaidi Chair of Research in Rheumatic Diseases, during the conduct of the study. The authors report no other conflicts of interest in this work.

\section{References}

1. Bikbov B, Purcell CA, Levey AS, et al. Global, regional, and national burden of chronic kidney disease, 1990-2017: a systematic analysis for the Global Burden of Disease Study 2017. Lancet. 2020;395 (10225):709-733. doi:10.1016/S0140-6736(20)30045-3
2. Hill NR, Fatoba ST, Oke JL, et al. Global prevalence of chronic kidney disease - A systematic Review and meta-analysis. PLoS One. 2016;11(7):e0158765. doi:10.1371/journal.pone.0158765

3. Becker BF. Towards the physiological function of uric acid. Free Radic Biol Med. 1993;14(6):615-631. doi:10.1016/0891-5849(93) 90143-I

4. de Cosmo S, Viazzi F, Pacilli A, et al. Serum uric acid and risk of CKD in type 2 diabetes. Clin J Am Soc Nephrol. 2015;10 (11):1921-1929. doi:10.2215/CJN.03140315

5. Obermayr RP, Temml C, Gutjahr G, Knechtelsdorfer M, Oberbauer R, Klauser-Braun R. Elevated uric acid increases the risk for kidney disease. $J$ Am Soc Nephrol. 2008;19(12):2407-2413. doi:10.1681/ASN.2008010080

6. Jalal DI, Chonchol M, Chen W, Targher G. Uric acid as a target of therapy in CKD. Am J Kidney Dis. 2013;61(1):134-146. doi:10.1053/ j.ajkd.2012.07.021

7. Li L, Yang C, Zhao Y, Zeng X, Liu F, Fu P. Is hyperuricemia an independent risk factor for new-onset chronic kidney disease?: a systematic review and meta-analysis based on observational cohort studies. BMC Nephrol. 2014;15(1):1-12. doi:10.1186/1471-2369-15122

8. Pizarro MH, Santos DC, Barros BSV, De Melo LGN, Gomes MB Serum uric acid and renal function in patients with type 1 diabetes: a nationwide study in Brazil. Diabetol Metab Syndr. 2018;10(1):22. doi:10.1186/s13098-018-0324-7

9. Srivastava A, Kaze AD, McMullan CJ, Isakova T, Waikar SS. Uric acid and the risks of kidney failure and death in individuals with CKD. Am J Kidney Dis. 2018;71(3):362-370. doi:10.1053/j.ajkd.20 17.08.017

10. Bartáková V, Kuricová K, Pácal L, et al. Hyperuricemia contributes to the faster progression of diabetic kidney disease in type 2 diabetes mellitus. J Diabetes Complications. 2016;30(7):1300-1307. doi:10. 1016/j.jdiacomp.2016.06.002

11. Chang Y-H, Lei -C-C, Lin K-C, Chang D-M, Hsieh C-H, Lee Y-J. Serum uric acid level as an indicator for CKD regression and progression in patients with type 2 diabetes mellitus-a 4.6-year cohort study. Diabetes Metab Res Rev. 2016;32(6):557-564. doi:10.1002/ dmrr.2768

12. Iseki $\mathrm{K}$, Ikemiya $\mathrm{Y}$, Inoue $\mathrm{T}$, Iseki $\mathrm{C}$, Kinjo $\mathrm{K}$, Takishita $\mathrm{S}$. Significance of hyperuricemia as a risk factor for developing ESRD in a screened cohort. Am J Kidney Dis. 2004;44(4):642-650. doi:10. 1053/j.ajkd.2004.06.006

13. Hsu CY, Iribarren C, McCulloch CE, Darbinian J, Go AS. Risk factors for end-stage renal disease - 25-year follow-up. Arch Intern Med. 2009;169(4):342-350. doi:10.1001/archinternmed.2008.605

14. Barkas F, Elisaf M, Liberopoulos E, Kalaitzidis R, Liamis G. Uric acid and incident chronic kidney disease in dyslipidemic individuals. Curr Med Res Opin. 2018;34(7):1193-1199. doi:10.1080/030079 95.2017.1372157

15. Takae K, Nagata M, Hata J, et al. Serum uric acid as a risk factor for chronic kidney disease in a Japanese Community- the Hisayama Study. Circ J. 2016;80(8):1857-1862. doi:10.1253/circj.CJ-16-0030

16. Badve SV, Pascoe EM, Tiku A, et al. Effects of allopurinol on the progression of chronic kidney disease. $N$ Engl J Med. 2020;382 (26):2504-2513. doi:10.1056/NEJMoa1915833

17. Afkarian M, Polsky S, Parsa A, et al. Preventing Early Renal Loss in Diabetes (PERL) study: a randomized double-blinded trial of allopurinoldrationale, design, and baseline data. Diabetes Care. 2019;42 (8):1454-1463. doi:10.2337/dc19-0342

18. Yang C-Y, Chen C-H, Deng S-T, et al. Allopurinol Use and risk of fatal hypersensitivity reactions. JAMA Intern Med. 2015;175(9):1550. doi:10.1001/jamainternmed.2015.3536

19. Frey N, Jossi J, Bodmer M, et al. The epidemiology of stevens-johnson syndrome and toxic epidermal necrolysis in the UK. J Invest Dermatol. 2017;137(6):1240-1247. doi:10.1016/j.jid.20 17.01.031 
20. Bardin T, Richette P. Definition of hyperuricemia and gouty conditions. Curr Opin Rheumatol. 2014;26(2):186-191. doi:10.10 97/BOR.0000000000000028

21. Eleftheriadis T, Golphinopoulos S, Pissas G, Stefanidis I. Asymptomatic hyperuricemia and chronic kidney disease: narrative review of a treatment controversial. J Adv Res. 2017;8(5):555-560. doi:10.1016/j.jare.2017.05.001

22. Fessel WJ. Renal outcomes of gout and hyperuricemia. Am J Med. 1979;67(1):74-82. doi:10.1016/0002-9343(79)90076-7

23. Kumagai T, Ota T, Tamura Y, Chang WX, Shibata S, Uchida S. Time to target uric acid to retard CKD progression. Clin Exp Nephrol. 2017;21(2):182-192. doi:10.1007/s10157-016-1288-2

24. Kohagura K, Tana T, Higa A, et al. Effects of xanthine oxidase inhibitors on renal function and blood pressure in hypertensive patients with hyperuricemia. Hypertens Res. 2016;39(8):593-597. doi: $10.1038 / \mathrm{hr} .2016 .37$

25. Chonchol M, Shlipak MG, Katz R, et al. Relationship of uric acid with progression of kidney disease. Am J Kidney Dis. 2007;50 (2):239-247. doi:10.1053/j.ajkd.2007.05.013

26. Madero M, Sarnak MJ, Wang X, et al. Uric acid and long-term outcomes in CKD. Am J Kidney Dis. 2009;53(5):796-803. doi:10. 1053/j.ajkd.2008.12.021

27. Zhu Y, Pandya BJ, Choi HK. Comorbidities of gout and hyperuricemia in the US general population: NHANES 2007-2008. Am J Med. 2012;125(7):679-687.e1. doi:10.1016/j.amjmed.2011.09.033

28. Sturm G, Kollerits B, Neyer U, Ritz E, Kronenberg F. Uric acid as a risk factor for progression of non-diabetic chronic kidney disease? The Mild to Moderate Kidney Disease (MMKD) Study. Exp Gerontol. 2008;43(4):347-352. doi:10.1016/j.exger.2008.01.006

29. Yu M-A, Sánchez-Lozada LG, Johnson RJ, Kang D-H. Oxidative stress with an activation of the renin-angiotensin system in human vascular endothelial cells as a novel mechanism of uric acid-induced endothelial dysfunction. J Hypertens. 2010;28(6):1. doi:10.1097/ HJH.0b013e328337da1d
30. Choi Y, Yoon Y, Lee K, et al. Uric acid induces endothelial dysfunction by vascular insulin resistance associated with the impairment of nitric oxide synthesis. FASEB J. 2014;28(7):3197-3204. doi:10.1096/ fj. 13-247148

31. Mazzali M, Hughes J, Kim Y-G, et al. Elevated uric acid increases blood pressure in the rat by a novel crystal-independent mechanism. Hypertension. 2001;38(5):1101-1106. doi:10.1161/hy1101.092839

32. Alem MM. Allopurinol and endothelial function: a systematic review with meta-analysis of randomized controlled trials. Cardiovasc Ther. 2018;36(4):12432. doi:10.1111/1755-5922.12432

33. Bose B, Badve SV, Hiremath SS, et al. Effects of uric acid-lowering therapy on renal outcomes: a systematic review and meta-analysis. Nephrol Dial Transplant. 2014;29(2):406-413. doi:10.1093/ndt/ gft378

34. Kanji T, Gandhi M, Clase CM, Yang R. Urate lowering therapy to improve renal outcomes in patients with chronic kidney disease: systematic review and meta-analysis. BMC Nephrol. 2015;16(1):58. doi:10.1186/s12882-015-0047-z

35. Su X, Xu B, Yan B, Qiao X, Wang L. Effects of uric acid-lowering therapy in patients with chronic kidney disease: a meta-analysis. PLoS One. 2017;12(11):e0187550. doi:10.1371/journal.pone.0187 550

36. Kimura K, Hosoya T, Uchida S, et al. Febuxostat therapy for patients with stage $3 \mathrm{CKD}$ and asymptomatic hyperuricemia: a Randomized Trial. Am J Kidney Dis. 2018;72(6):798-810. doi:10.1053/j.ajkd.20 18.06.028

37. Doria A, Galecki AT, Spino C, et al. Serum urate lowering with allopurinol and kidney function in type 1 diabetes. $N$ Engl $\mathrm{J} \mathrm{Med}$. 2020;382(26):2493-2503. doi:10.1056/NEJMoa1916624

38. Feig DI. Urate-lowering therapy and chronic kidney disease progression. N Engl J Med. 2020;382(26):2567-2568. doi:10.1056/ NEJMe2015886
International Journal of General Medicine

\section{Publish your work in this journal}

The International Journal of General Medicine is an international, peer-reviewed open-access journal that focuses on general and internal medicine, pathogenesis, epidemiology, diagnosis, monitoring and treatment protocols. The journal is characterized by the rapid reporting of reviews, original research and clinical studies across all disease areas. The manuscript management system is completely online and includes a very quick and fair peer-review system, which is all easy to use. Visit http://www.dovepress.com/ testimonials.php to read real quotes from published authors. 